\title{
Avanços e desafios em normatização de amostras grátis de medicamentos no Brasil
}

\author{
I ${ }^{1}$ Carla Patricia F. A. de Souza, ${ }^{2}$ Jaime L. M. Oliveira, ${ }^{3}$ Débora C. Kligerman I
}

Resumo: Este trabalho analisou criticamente a legislação sobre amostras grátis de medicamentos no Brasil, pontuando alguns aspectos importantes relacionados à saúde da população. Foi realizado um levantamento das leis, decretos, normas e regulamentações que norteiam o assunto. A amostra grátis é uma das estratégias de publicidade e marketing usadas pela indústria farmacêutica. Em alguns casos, a indicação de certos medicamentos é baseada em dados técnicos apresentados pelos propagandistas. Por outro lado, os médicos que buscam informaçôes técnicas em fontes com menor influência da indústria farmacêutica indicam menos os medicamentos novos, que na maioria das vezes são mais caros. Embora tenha sido observado um avanço na normatização em relação às amostras grátis, ela ainda é incipiente. Tópicos como o prazo mínimo para sua distribuição, quantidade máxima a ser entregue a cada prescritor, seu transporte, armazenamento e cuidados com o prazo de validade deveriam ser incluídas na legislação pertinente, como ocorre em outros países. Estas discussões deveriam ser retomadas com a menor influência possível dos laboratórios farmacêuticos, seguindo critérios técnico-científicos. Portanto, é necessário que este assunto seja contextualizado nas resoluções sobre amostras grátis de medicamentos, seguindo as normas das boas práticas de fabricação.

> Palavras-chave: legislação de medicamentos; amostra grátis de medicamentos; publicidade na indústria farmacêutica; propaganda de medicamentos.

\author{
1 Departamento de Saneamento \\ e Saúde Ambiental, Escola \\ Nacional de Saúde Pública \\ Sérgio Arouca, Fundação \\ Oswaldo Cruz. Rio de Janeiro- \\ RJ, Brasil. cpatriciafigueiredo@ \\ bol.com.br \\ ${ }^{2}$ Departamento de Saneamento \\ e Saúde Ambiental, Escola \\ Nacional de Saúde Pública \\ Sérgio Arouca, Fundação \\ Oswaldo Cruz. Rio de Janeiro- \\ RJ, Brasil. Endereço eletrônico: \\ jaimel@ensp.fiocruz.br \\ ${ }^{3}$ Departamento de Saneamento \\ e Saúde Ambiental, Escola \\ Nacional de Saúde Pública, \\ Fundação Oswaldo Cruz. \\ Rio de Janeiro-RJ, Brasil. \\ deboracyklig@gmail.com
}


É indiscutível que um dos reflexos da evolução das políticas de saúde ao longo dos anos foi a preocupação cada vez mais significativa no que se refere ao fabrico e comercialização de medicamentos e produtos para a saúde. Nesse contexto, o medicamento passou a ser considerado elemento essencial na prevenção e recuperação da saúde. Consequentemente, foi criada a Política Nacional de Medicamentos, que tem como objetivo principal assegurar o acesso da população a medicamentos seguros, eficazes e de qualidade, ao menor custo possível. De acordo com esta política, o Ministério da Saúde fica responsável pela elaboração da Relação Nacional de Medicamentos Essenciais, na qual constam produtos considerados básicos e indispensáveis para atender à maioria dos problemas de saúde da população. Portanto, esta relação passa ser a base para a organização das listas estaduais e municipais de medicamentos no Brasil (BRASIL, 2001).

A produção de medicamentos adotados pelo sistema público de saúde é sustentada por quatro pilares fundamentais: segurança, eficácia, acesso e qualidade. Como a garantia ao acesso igualitário a medicamentos está preconizada pelos princípios do Sistema Único de Saúde (SUS), o Estado brasileiro se tornou o maior consumidor de medicamentos. Logo, o sistema de qualidade adotado pelas Boas Práticas de Fabricação de medicamentos - BPF (BRASIL, 2010) passou a ser um dos norteadores das ações da Vigilância Sanitária no setor de medicamentos em nosso país, sendo incorporado a sua regulamentação como condição necessária para a comercialização de produtos (DEUS; SÁ, 2011).

Segundo Cassiani (2005), cerca de 90\% dos pacientes que procuram os serviços profissionais médicos recebem prescrições de medicamentos. Contudo, para que o uso de medicamentos seja considerado uma alternativa efetiva, além da sua utilização orientada, eles devem cumprir critérios mínimos de qualidade.

A parceria entre a indústria farmacêutica e a classe médica foi intensificada nas últimas décadas, trazendo à tona situações com enorme potencial de conflitos de interesses, as quais são atualmente tema de estudo de diferentes áreas do conhecimento. Dentre essas situações, a propaganda de medicamentos pode influenciar no julgamento ou decisão de um profissional em suas atividades administrativas, gerenciais, de ensino e de pesquisa (COSTA-VAL, 2007; MARQUES FILHO, 2010). Desse modo, a indústria farmacêutica realiza altos investimentos na publicidade de seus produtos, destacando-se as amostras 
grátis de medicamentos. Normalmente, esse investimento é pago indiretamente

pelo incremento das vendas, contribuindo para que o setor esteja entre os mais lucrativos do mundo (FIASCHETTI, 2009). Portanto, há crescente preocupação com a influência negativa do marketing utilizado pela indústria sobre o padrão de consumo e prescrição de medicamentos, principalmente das autoridades de saúde, agências regulatórias e profissionais da área da Saúde Pública (BARROS, 2000; SILVA; JUNGES, 2010).

A Lei no 6.360 (BRASIL, 1976) foi um dos marcos na regulamentação de medicamentos, inclusive por iniciar uma abordagem sobre a questão da fiscalização de sua propaganda. No final da década de 1990, a Agência Nacional de Vigilância Sanitária (ANVISA) publicou a RDC no 102 (BRASIL, 2000), que regulamentou a publicidade de medicamentos, mas foi só em 2008, com a RDC no 96 (BRASIL, 2008) que se realizou uma abordagem mais detalhada sobre amostra grátis de medicamento (BRASIL, 2009; DEUS; SÁ, 2011).

A RDC no 60 (BRASIL, 2009) norteia o uso e distribuição de amostras grátis de medicamentos, mas muitas questôes ainda precisam ser discutidas e regulamentadas. Tal norma não define a quantidade de amostras grátis que podem ser entregues, bem como o prazo mínimo autorizado para sua distribuição. Também aborda de forma superficial os mecanismos de transporte, armazenamento e controle de distribuição desses materiais. As lacunas não contempladas nessa norma podem afetar diretamente a saúde da população (LEITÃO; SIMÔES; FRANÇA, 2012).

Este estudo tem como objetivo analisar a normatização da amostra grátis de medicamentos no Brasil e discutir pontos importantes não contemplados pela legislação.

\section{Medicamentos no contexto das políticas de saúde no Brasil}

A unificação do sistema de saúde e sua descentralização para estados e municípios como uma política de saúde pública visam promover a universalização do atendimento e equalização do acesso com extensão de cobertura de serviços, aumentar a participação da população através de entidades representativas na formulação, gestão, execução e avaliação das políticas e ações de saúde (controle social) e racionalizar e otimizar os recursos setoriais com financiamento do Estado através de um Fundo Único de Saúde em nível federal (DEUS; SÁ, 2011). 
Embora o medicamento seja um dos componentes fundamentais da atenção à saúde, seu uso inadequado pode causar mais prejuízos do que benefícios (BRASIL, 2001). Logo, o uso racional de medicamentos constitui uma das estratégias para alcançar esse modelo de saúde através da mobilização de recursos e esforços do Estado (HUNT; KHOSLA, 2008).

Na década de 1990, as instâncias gestoras e de controle social do país passaram a avaliar e regulamentar a ampliação do acesso aos medicamentos. No Brasil, dada a necessidade de criação e instituição de políticas públicas que esclarecessem e regulassem esse acesso, foi consolidada a Política Nacional de Medicamentos (PNM) em 1998. Essa política é parte essencial da Política Nacional de Saúde e constitui um dos elementos fundamentais para a efetiva implementação de ações capazes de promover a melhoria das condições da assistência à saúde da população. Uma de suas diretrizes é o desenvolvimento de atividades relacionadas à promoção do acesso da população aos medicamentos essenciais (BRASIL, 2001). As ações para alcançar esses propósitos incluem a regulamentação sanitária dos medicamentos, a promoção do uso racional, bem como a reorientação da assistência farmacêutica, o desenvolvimento científico e tecnológico, a promoção da produção de medicamentos e a garantia da segurança, eficácia e qualidade dos medicamentos (FALQUETO; KLIGERMAN, 2007).

\section{Publicidade e propaganda de medicamentos}

A propaganda é reconhecida como um processo de produção de formas culturais e visível de representação de identidades, já que em seu discurso afluem diferentes figuras do imaginário sociocultural (CASTRO, 2006). No passado, a circulação dos anúncios era limitada ao público leitor dos jornais. Neste universo, os laboratórios e indústrias farmacêuticas passaram a ver nos meios de comunicação de grande massa oportunidade de aumentar seus lucros. Assim, foram criadas inúmeras estratégias para reter a atenção dos leitores, dando ênfase aos testemunhos dos benefícios alcançados à saúde pelos medicamentos, provocando descontos e popularizando o anúncio em cores (CUNHA; NASCIMENTO, 2008).

Ainda no século XIX, tudo que fosse levado aos chamados "reclames", que eram mantidos pelo setor farmacêutico, tinha cura, inclusive as denominadas "dores da alma”. Os anúncios divulgavam que os remédios poderiam prevenir até os divórcios e os suicídios. Já na década de 1940, era comum “as divas da 
beleza” convencerem sobre o hábito de consumir calmantes. Os almanaques de farmácia popularizaram os medicamentos e os artigos de cosméticos no Brasil. No começo eles eram restritos às antigas farmácias de manipulação, vindo posteriormente a fazer parte das estratégias publicitárias de grandes laboratórios químico-farmacêuticos (GOMES, 2006).

Durante as primeiras décadas do século XX, a automedicação era bastante estimulada pela propaganda de medicamentos. Nessa época, os reguladores menstruais, fortificantes, tônicos, depurativos, purgantes, analgésicos e antiácidos eram os mais consumidos (BRASIL, 2008). Do anúncio puro e simples, as empresas passaram a desenvolver estratégias na qual publicidade, propaganda e açôes de mercado estavam articuladas para assegurar o sucesso das vendas (BRASIL, 2009). A indústria farmacêutica passou a utilizar diversos recursos promocionais direcionados aos profissionais médicos, visando a construção de uma opinião favorável em relação a seus produtos, bem como a divulgação institucional do próprio laboratório (FIASCHETTI, 2009). Desse modo, o setor passou a colocar anúncios em revistas médicas e publicações técnicas, patrocinar e/ou organizar simpósios, congressos e eventos ligados à saúde, promover eventos sociais, pagamento de refeições, subsídios de viagens e hospedagens, distribuir brindes e materiais promocionais e publicar dicionários e suplementos terapêuticos (MIGUELOTE; CAMARGO JR, 2010). Dentre os mecanismos mais bem-sucedidos, o setor passou a investir pesado na distribuição de amostras grátis de medicamento. A estratégia adotou visitas técnicas por representantes da empresa (propagandistas), que costumam deixar as amostras como forma de marketing do laboratório farmacêutico (BREEN, 2004). O resultado deste investimento se reflete nas altas vendas nas farmácias e drogarias.

A propaganda exerce papel importante no uso de medicamentos, por influenciar diretamente prescritores e usuários. Essa influência, embora quase nunca reconhecida pelos médicos, interfere na prescrição, que passa a ser guiada por outras finalidades que não a terapêutica (BARROS; JOANY, 2012). Fiaschetti (2009) fez uma comparação entre médicos que buscam as informações técnicas apenas na propaganda da indústria farmacêutica com aqueles que se atualizavam através de educação permanente e participação em eventos científicos. Os resultados mostraram que os médicos que utilizam como principal fonte de informação os materiais promocionais dos laboratórios ficam propensos à adoção 
de novos medicamentos; já aqueles que buscam outras fontes de informaçōes foram os que prescreveram de forma mais racional. Vale ressaltar que os novos medicamentos podem ter maior potencial de reaçóes adversas indesejadas e normalmente desconhecidas, além do seu alto custo (FIASCHETTI, 2009).

Segundo Jones e colaboradores (2001), os médicos não especialistas são os que mais prescrevem os lançamentos da indústria farmacêutica e para uma maior gama de condições. Estes profissionais normalmente se baseiam em informações disponibilizadas pelos laboratórios, especialmente pelos propagandistas. Por outro lado, os médicos especialistas tendem a prescrever menos os novos medicamentos, pautando suas decisōes preferencialmente em artigos e reuniōes científicas (JONES; GREENFIELD; BRADLEY, 2001). Portanto, estudos e esforços devem ser empreendidos para uma melhor compreensão da percepção e das atitudes dos médicos frente às ações promocionais da indústria farmacêutica. Além disso, é necessário fortalecer os programas de treinamento aos profissionais prescritores, bem como melhor divulgar as fontes que possuem menor influência dos laboratórios farmacêuticos a respeito de informaçōes técnicas sobre medicamentos, tais como o Portal da Saúde Baseada em Evidências, do Ministério da Saúde. Assim, os médicos prescritores podem ser mais bem esclarecidos sobre a eficácia dos medicamentos, principalmente os novos. Essas medidas poderão reduzir a influência do propagandista na prescrição dos medicamentos.

\section{Legislação de amostra grátis de medicamentos}

ALei no 6.360 (BRASIL, 1976) ficou conhecida como a Lei de Vigilância Sanitária e contribuiu para reforçar as exigências legais nas mais diversas áreas, inclusive sobre a propaganda de medicamentos. No ano seguinte, o Decreto no 79.094 (BRASIL, 1977), além de criar a Secretaria Nacional de Vigilância Sanitária (SNVS), introduziu pontos importantes, como a necessidade de autorização prévia pelo Ministério da Saúde para anúncios de produtos sujeitos à vigilância sanitária e a proibição de propaganda de medicamentos vendidos sob prescrição médica (BRASIL, 1977). O Código de Defesa do Consumidor (BRASIL, 1990) passou a responsabilizar o produtor pela qualidade de seus produtos e serviços. Em relação à vigilância sanitária, este Código reforçou a legislação específica de proteção e defesa da saúde (BUENO; TAITELBAU, 2008). 
Ainda na mesma década, o Brasil passou por um processo drástico de reformas do aparelho do Estado, dentre eles a criação da ANVISA, que alterou o arranjo de vigilância sanitária no país (LUCCHESE, 2008). Entre outras metas, esta agência visa à instalação de mecanismos efetivos para regulamentar e fiscalizar a propaganda de medicamentos, com o objetivo de buscar a proteção e a promoção da saúde da população, de modo a garantir a segurança sanitária de produtos e serviços. Já no primeiro ano de sua criação, foi elaborada uma proposta de regulamentação da publicidade e propaganda de medicamentos consolidando a RDC no 102 (BRASIL, 2000). Esta resolução teve importante papel no controle da promoção e divulgação de medicamentos de produção nacional ou importados. Antes, informações como contraindicações, indicações, precauções, cuidados e advertências estavam presentes apenas em $28 \%$ das propagandas. Portanto, essa norma representou um marco histórico para a consolidação da atuação da ANVISA como elemento articulador das políticas públicas de intervenção e proteção da sociedade brasileira (FAGUNDES et al., 2007).

Em 2002, a ANVISA implantou junto às universidades o Projeto de Monitoração da Propaganda e Publicidade de Medicamentos, que demonstrou a necessidade de atualizar a RDC no 102 (BRASIL, 2000). Após quatro anos de discussão, surgiu uma proposta do novo regulamento que gerou grande repercussão na época (ARAUJO; BOCHNER; NASCIMENTO, 2012). Como resultado, foi publicada a RDC no 96 (BRASIL, 2008), ainda vigente, cujo objetivo é regulamentar a divulgação e promoção comercial de medicamentos (ARAUJO; BOCHNER; NASCIMENTO, 2012). Em relação às amostras grátis de medicamentos, a RDC no $102 / 2000$ possuía um único artigo com três parágrafos, enquanto que a nova resolução aborda o mesmo assunto em um título com três artigos. No entanto, não existe diferença profunda entre as duas resoluções sobre este assunto, exceto sobre a proibição de amostras grátis de vacinas e sobre a especificidade dos antibióticos e anticoncepcionais (BRASIL, 2000; 2008). Vale ressaltar a maior importância dada na redação da nova norma às questôes relativas à visita de propagandistas e patrocínio a eventos científicos pelos laboratórios farmacêuticos, inclusive nas campanhas sociais (BRASIL, 2008). Os itens que eram abordados em um único título pela RDC no 102 (BRASIL, 2000) foram distribuídos e mais bem elaborados em cinco capítulos distintos e específicos na RDC no 96 sobre propaganda e publicidade de medicamentos (BRASIL, 2008). 
Na proposta inicial para a RDC no 96 (BRASIL, 2008), havia a obrigação de distribuição das amostras grátis somente aos médicos prescritores com o prazo máximo de dois anos após seu registro, como ocorre nos países europeus (ESPANHA, 1994; PORTUGAL, 2008). Além disso, constavam a proibição da distribuição gratuita de nova apresentação de medicamentos já existentes e a exigência de que as amostras grátis deveriam ter $100 \%$ do conteúdo da embalagem original dos medicamentos prescritos e $50 \%$ dos isentos de prescrição (BRASIL, 2005). No entanto, a classe médica e a indústria farmacêutica foram contrárias a essas imposições. No ano seguinte, foi elaborada uma nova resolução específica sobre a produção, dispensação e controle de amostras grátis de medicamentos - a RDC no 60 (BRASIL, 2009). A nova regulamentação elucida que as amostras grátis devem seguir os padrões de fabricação e embalagens dos produtos originais registrados na ANVISA, apresentando os mesmos instrumentos de rastreabilidade e autenticidade. Segundo a ANVISA (BRASIL, 2013), essas medidas permitem o rastreamento do medicamento desde sua produção até a prescrição e a segurança na embalagem, garantindo sua identificação. Além disso, os prescritores passam a ser responsáveis pela conservação e validade do estoque das amostras. No caso dos antibióticos e anticoncepcionais, os profissionais deverão entregar ao paciente a quantidade suficiente para o completo tratamento (BRASIL, 2009).

Embora tenha havido uma evolução normativa em relação às amostras grátis de medicamentos, os itens conflitantes não foram atendidos. A ANVISA, como o órgão regulador competente que visa implementar as ações de promoção e segurança da saúde pública no Brasil, deveria rever os conceitos de amostra grátis com menor influência das pressôes dos laboratórios farmacêuticos. Assim, questôes como a necessidade de maior controle da quantidade de amostra que é efetivamente entregue aos prescritores, bem como o prazo de distribuição após seu registro, deveriam ser regulamentadas como ocorre nos países europeus.

O Real Decreto no 1.416/1994 (ESPANHA, 1994) e a Deliberação no 44/ CD/2008 (PORTUGAL, 2008) condicionam um limite máximo de amostras grátis a serem distribuídas por ano a cada prescritor e definem que somente após dois anos do seu registro será permitida sua entrega gratuita. Além disso, as normas brasileiras vigentes não exigem que o transporte e armazenamento das amostras grátis de medicamentos sigam as mesmas exigências das Boas 
Práticas de Fabricação de Medicamentos, como podem ser encontradas nas regulamentações internacionais.

\section{Considerações finais}

O medicamento é um componente fundamental na promoção de saúde pública. As amostras grátis de medicamentos são um importante veículo de propaganda da indústria farmacêutica e precisam ser contextualizadas na legislação de medicamentos e não somente nas de publicidade e propaganda. Somente assim será possível ampliar e harmonizar os mecanismos de proteção à saúde da população.

Alguns médicos baseiam suas prescrições em informações técnicas dos propagandistas. A influência do mercado pode comprometer os objetivos da Política Nacional de Medicamentos em relação a sua segurança e eficácia a um menor custo, porque esse mecanismo de marketing empresarial favorece os novos lançamentos dos laboratórios, que são na maioria dos casos mais caros. Seria importante haver maior incentivo da participação ativa dos profissionais prescritores em cursos de atualização e capacitações, para ampliar seu conhecimento sobre a ação dos medicamentos, principalmente os novos. Além disso, deveria haver melhor divulgação das fontes disponibilizadas pelo governo em relação às informaçôes sobre os medicamentos, isentas da influência da indústria farmacêutica.

Embora a normatização de amostra grátis de medicamento no Brasil tenha conseguido alguns avanços importantes, como a publicação da RDC no 60 (BRASIL, 2009), alguns tópicos importantes ainda precisam ser revistos e incluídos. Portanto, esse assunto precisa ser mais explorado, para garantir que a distribuição e o uso desses medicamentos sejam realizados com maior segurança e efetividade. ${ }^{1}$

\section{Referências}

ARAUJO, C.P.; BOCHNER, R.; NASCIMENTO, A.C. Marcos legais da propaganda de medicamentos: avanços e retrocessos. Physis: Revista de Saúde Coletiva, Rio de Janeiro, v. 22, p. 331-346, 2012.

BARROS, J.A.C. A (Des)informação sobre medicamentos: o duplo padrão de conduta das empresas farmacêuticas. Cadernos de Saúde Pública, Rio de Janeiro, v. 16, n. 2, p. 421-427, 2000. 
BARROS, J.A.C.; JOANY, S. Anúncios de medicamentos em revistas médicas: ajudando a promover a boa prescrição? Ciência \& Saúde Coletiva, Recife, v. 7, n. 4, p. 891-898, 2002.

BRASIL. Ministério da Saúde. Agência Nacional de Vigilância Sanitária. Análise do Consolidado de propostas encaminhadas para a Consulta no 84/2005. Disponível em: http:// portal.anvisa.gov.br/wps/portal/anvisa/home. Acesso em: 23 jul 2013.

BRASIL. Ministério da Saúde. Agência Nacional de Vigilância Sanitária. Consulta pública sobre rastreabilidade de medicamentos no 10/2013. Disponível em: http://portal.anvisa. gov.br/wps/portal/anvisa/home. Acesso em: 30 ago 2013.

BRASIL. Ministério da Saúde. Agência Nacional de Vigilância Sanitária. Resolução RDC $n^{\circ} 102$, de 30 de novembro de 2000. Aprova o regulamento sobre propagandas, mensagens publicitárias e promocionais e outras práticas de divulgação, promoção ou comercialização de medicamentos. Disponível em: http://www.fenapro.org.br/legislacao/download/ resol_102_00rdc.pdf. Acesso em: 15 jul 2013.

BRASIL. Ministério da Saúde. Agência Nacional de Vigilância Sanitária. Resolução RDC $n^{\circ}$ 96, de 17 de dezembro de 2008. Dispõe sobre a propaganda, publicidade, informação e outras práticas cujo objetivo seja a divulgação ou promoção comercial de medicamentos. Disponível em: http://www.anvisa.gov.br/propaganda/rdc/rdc_96_2008_consolidada.pdf. Acesso em: 13 jul 2013.

BRASIL. Ministério da Saúde. Agência Nacional de Vigilância Sanitária. Resolução RDC $n^{\circ}$ 60, de 26 de novembro de 2009. Dispõe sobre a produção, dispensação e controle de amostras grátis de medicamentos e dá outras providências. Disponível em: http://brasilsus. com.br/legislacoes/rdc/101526-60. Acesso em: 7 jul 2013.

BRASIL. Ministério da Saúde. Agência Nacional de Vigilância Sanitária. Resolução $R D C n^{o} 17$, de 16 de abril de 2010. Dispõe sobre as Boas Práticas de Fabricação de Medicamentos. Disponível em: http://bvsms.saude.gov.br/bvs/saudelegis/anvisa/2010/ res0017_16_04_2010.html. Acesso em: 10 ago 2013.

BRASIL. Lei nº 6.360, de 23 de setembro de 1976. Lei de Vigilância Sanitária. Dispõe sobre a vigilância sanitária a que ficam sujeitos os medicamentos, as drogas, os insumos farmacêuticos e correlatos, cosméticos, saneantes e outros produtos, e dá outras providências. Diário Oficial da República Federativa do Brasil, Poder Executivo, Brasília, Distrito Federal. Seção 1, p. 12.647. 1976.

BRASIL. Decreto n. 79.094, de 5 de janeiro de 1977. Regulamenta a Lei no 6.360, de 23 de setembro de 1976, que submete a sistema de vigilância sanitária os medicamentos, insumos farmacêuticos, drogas, correlatos, cosméticos, produtos de higiene, saneantes e outros. Diário Oficial da República Federativa do Brasil, Poder Executivo, Brasília, Distrito Federal, 07 jan 1977, p.11; revogada pelo Decreto no 8.077, de 14 de agosto de 2013. Seção 1, p.18. 
BRASIL. Lei no 8.078, de 11 de setembro de 1990. Dispõe sobre a proteção do consumidor e dá outras providências. Diário Oficial da União, Brasília, Distrito Federal. Disponível em: http://www.planalto.gov.br/ccivil_03/leis/18078.htm. Acesso em: 02 ago 2013.

BRASIL. Ministério da Saúde. Secretaria de Políticas de Saúde. Política Nacional de Medicamentos. Brasília: Ministério da Saúde, 2001 (Série C - Projetos, Programas e Relatórios, n. 25).

BRASIL. Ministério da Saúde. Secretaria de Políticas de Saúde. Departamento de Atenção Básica. Incentivo à Assistência Farmacêutica Básica: o que é e como funciona. Brasília: Ministério da Saúde, 2001.

BREEN, K.J. The medical profession and the pharmaceutical industry: when will we open our eyes? Medical Journal of Australia. Sydney, v. 180, p. 409-410, 2004.

BUENO, E.; TAITELBAU, M.P. Vendendo Saúde: a história da propaganda de medicamentos no Brasil. Brasília: ANVISA, 2008.

CASSIANI, S.H.B. A segurança do paciente e o paradoxo no uso de medicamentos. Revista Brasileira de Enfermagem. Brasília, v. 58, n. 1, p. 95-99, 2005.

CASTRO, M.H. Caminhos cruzados entre a propaganda e a saúde em 1930. Revista Fronteiras: Estudos Midiáticos VIII. Porto Alegre, v. 8, n.2, p. 203-211, 2006.

COSTA-VAL, R. A Iminente mudança de paradigma na relação entre a indústria farmacêutica e a medicina. Jornal Vascular Brasileiro. São Paulo, v. 6, n. 1, p. 104-106, 2007. CUNHA, S.R.M.; NASCIMENTO, L.M. Imagem da saúde: a medicalização da mulher em propagandas de fármacos do início do século XX. In: NASCIMENTO, L.; LOPES, C.M.; CHAVES, L.M.N. (Orgs.). Saúde, linguagem e imaginário. v. 2, Rio Branco: EdEDUFAC, 2008. 30 p.

DEUS, F.J.T.; SÁ, P.F.G. Evolução da normatização de boas práticas de fabricação (BPF) e o seu impacto na qualidade de medicamentos comercializados no Brasil. In: MOSTRA DE PRODUÇÃO CIENTÍFICA DA PÓS-GRADUAÇÃO LATO SENSU DA PUC GOIÁS, 6., 2011.

ESPAÑA. Real Decreto 1416, de 25 de junio de 1994. Por el que se regula la publicidad de los medicamentos de uso humano. Barcelona. BOE n. 180. Disponível em: http://www. aemps.gob.es/legislacion/espana/otrosTemas/publicidad.htm. Acesso em: 20 out 2013.

FAGUNDES, M.J.D. et al. Análise bioética da propaganda e publicidade de medicamentos. Ciência \& Saúde Coletiva, Rio de Janeiro. v. 12, p. 221-229, 2007.

FALQUETO, E.; KLIGERMAN, D. C. Residuos relacionados à medicamentos sujeitos a controle especial pela vigilância sanitária: estudo de caso do diazepam utilizado no município de São Mateus-ES. 2007. 134 p. Dissertação (Mestrado em Saúde Pública) - Escola Nacional de Saúde Pública Sergio Arouca, Fundação Oswaldo Cruz, Rio de Janeiro, 2007. 
FIASCHETTI, M.A. Opiniōes e atitudes dos médicos frente às ações promocionais da indústria farmacêutica. 2009. Dissertação (Mestrado em Ciências Farmacêuticas) - Faculdade de Ciências Farmacêuticas, Universidade Estadual de São Paulo, São Paulo, 2009.

GOMES, M.L. Vendendo saúde! Revisitando os antigos almanaques de farmácia. Revista História, Ciência, Saúde - Manguinhos. Rio de Janeiro, v. 13, n. 4, p. 1.007-1.018, 2006.

HUNT, P.; KHOSLA, R. Acesso a medicamentos como um direito humano. SUR: Revista Internacional de Direitos Humanos. São Paulo, v. 8, p. 101, 2008.

JONES, M.I.; GREENFIELD, S.M.; BRADLEY, C.P. Prescribing new drugs: qualitative study of influences on consultants and general practitioners. British Medical Journal. Nova York, v. 323, p. 378-384, 2001.

LEITÃO, L.C.A.; SIMÕES, M.O.S.; FRANÇA, I.S.X. A saúde pública e a indústria farmacêutica: implicações bioéticas na produção do cuidado. Revista Brasileira de Ciências da Saúde. São Paulo, v. 16, p. 295-302, 2012.

LUCCHESE, G. Globalização e regulação sanitária: os rumos da vigilância sanitária no Brasil. Brasília: Anvisa, 2008.

MIGUELOTE, V.; CAMARGO JR., K.R. Indústria do conhecimento: uma poderosa engrenagem. Revista de Saúde Pública. São Paulo, v. 44, n. 1, p. 190-196, 2010.

MARQUES FILHO, J. A dimensão bioética dos conflitos de interesses na relação entre médico e indústria farmacêutica. Revista Brasileira de Clínica Médica. São Paulo, v. 8, n. 2, p. 148-53, 2010.

PORTUGAL. Deliberação no 44/CD, de 07 de fevereiro de 2008. Aprova o regulamento sobre os aspectos da publicidade de medicamentos previstos nos artigos $154^{\circ}, 162^{\circ} \mathrm{e}$ $202^{\circ}, n^{\circ} 1, j$, do Decreto-Lei no 176/2006, que constitui o anexo à presente deliberação e dela faz integrante. Lisboa. Disponível em: http://www.infarmed.pt/portal/page/portal/ INFARMED/LEGISLACAO/LEGISLACAO_FARMACEUTICA_COMPILADA/ TITULO_III/TITULO_III_CAPITULO_I/39-E_Delib_44_2008.pdf. Acesso em: 20 out 2013.

SILVA, A.S.; JUNGES, F. A evolução da regulamentação da propaganda e publicidade de medicamentos no Brasil: RDC 96/08 versus RDC 102/00. In: MOSTRA DE PRODUÇĀO CIENTÍFICA DA PÓS-GRADUAÇÃO LATO SENSU DA PUC GOIÁS, 5. 2010.

\section{Nota}

${ }^{1}$ C.P.F.A. Souza participou da concepção e redação do artigo; coleta, análise e interpretação dos dados. J.L.M. Oliveira e D.C. Kligerman participaram da redação e revisão crítica do conteúdo; aprovação da versão final a ser publicada. 


\section{Abstract}

Advances and challenges in standardization of free samples of drugs in Brazil

This paper critically analyzed the legislation on free samples of medicines in Brazil, scoring some important health-related aspects of the population. A survey of laws, decrees, rules and regulations that guide the subject was conducted. The free sample is one of the advertising and marketing strategies used by the pharmaceutical industry. In some cases, the designation of certain drugs is based on technical data provided by the propagandists. On the other hand, physicians seeking technical information on sources with less influence of the pharmaceutical industry indicate fewer new drugs, which most often are more expensive. Although an improvement in standardization has been seen for free samples, it is still incipient. Topics such as the time limit for its distribution, maximum to be delivered to each prescriber, transport, storage and care of the expiration date amount should be included in the relevant legislation, as in other countries. These discussions should be resumed with the least possible influence of pharmaceutical companies, following technical and scientific criteria. Therefore, it is necessary that this matter be contextualized in resolutions on free samples of medicines, following the standards of good manufacturing practices.

> Key words: drug legislation; free sample of drugs; pharmaceutical industry advertising; drug advertising. 